\title{
Structural characterization of Platanthera ussuriensis chloroplast genome and comparative analyses with other species of Orchidaceae
}

\author{
Chenyang Han ${ }^{1 \dagger}$, Rui Ding ${ }^{2 \dagger}$, Xiaoyan Zong ${ }^{1}$, Lijie Zhang ${ }^{3}$, Xuhui Chen ${ }^{1 *}$ and Bo Qu ${ }^{1}$
}

\begin{abstract}
Background: The genus Tulotis has been classified into the genus Platanthera in the present taxonomic studies since the morphological characteristics of this genus is very similar to that of Platanthera. Platanthera ussuriensis, formerly named as Tulotis ussuriensis, is a small terrestrial orchid species and has been listed as wild plant under State protection (category II) in China. An improved understanding of the genomic information will enable future applications of conservation strategy as well as phylogenetic studies for this rare orchid species. The objective of this research was to characterize and compare the chloroplast genome of P. ussuriensis with other closely related species of Orchidaceae.

Results: The chloroplast genome sequence of $P$. ussuriensis is 155,016 bp in length, which included a pair of inverted repeats (IRs) of 26,548 bp that separated a large single copy (LSC) region of 83,984 bp and a small single copy (SSC) region of $17,936 \mathrm{bp}$. The annotation contained a total of 132 genes, including 86 protein-coding genes, 38 tRNA genes and 8 rRNA genes. The simple sequence repeat (SSR) analysis showed that there were 104 SSRs in the chloroplast genome of $P$. ussuriensis. RNA editing sites recognition indicated 72 RNA editing events occurred, and all codon changes were $\mathrm{C}$ to $\mathrm{T}$ conversions. Comparative genomics showed that the chloroplast sequence of Platanthera related species were relatively conserved, while there were still some high variation regions that could be used as molecular markers. Moreover, Platanthera related species showed similar IR/SSC and IR/LSC borders. The phylogenetic analysis suggested that $P$. ussuriensis had a closer evolutionary relationship with P. japonica followed by the remaining Platanthera species.
\end{abstract}

Conclusion: Orchidaceae is a key group of biodiversity protection and also a hot spot group in the plant taxonomy and evolution studies due to their characteristics of high specialization and rapid evolution. This research determined the complete chloroplast genome of $P$. ussuriensis for the first time, and compared the sequence with other closely related orchid species. These results provide a foundation for future genomic and molecular evolution of the Orchidaceae species, and provide insights into the development of conservation strategy for Platanthera species.

Keywords: Platanthera ussuriensis, Orchidaceae, Chloroplast genome, Molecular evolution, Phylogenetic analysis, SSR markers, RNA editing site

\footnotetext{
*Correspondence: xhchen@syau.edu.cn

${ }^{\dagger}$ Chenyang Han and Rui Ding contributed equally to this work and are co-first authors.

${ }^{1}$ College of Bioscience and Biotechnology, Shenyang Agricultural University, Shenyang 110161, China

Full list of author information is available at the end of the article
}

\section{Background}

Orchidaceae is one of the largest family of angiosperms and also a key group of biodiversity protection $[1,2]$. The group of orchid species are widely distributed in almost 
all kinds of terrestrial ecosystems in the world, and is a hot spot group in the plant taxonomy and evolution studies due to the characteristics of high specialization and rapid evolution [3]. The classification system of orchid species was mainly based on morphological characteristics for a long time [4]. However, it is now widely recognized that the identification of orchid species is difficult, especially in the non-flowering period when many orchid species plants have very similar morphological characteristics. On the other hand, orchid flowers could be influenced by the selection pressure of their pollinators so easily that it is still difficult to distinguish them even at flowering stages. Moreover, many orchid species could crossbreed successfully in a wide range, resulting in many intermediate types and large natural variations. All of these make it very difficult for traditional taxonomy based on the morphological characteristics of orchids [59]. In terms of phylogeny, it is also difficult to understand the phylogenetic relationship between various groups because of the relatively complex evolutionary process and multi lineage origin of many orchid genera $[10,11]$.

As the photosynthetic organ in plants, chloroplast has an independent genome and the capacity of replication, transcription and translation [12]. The chloroplast genome of angiosperms usually consists of four parts and form a circular double chain structure including one large single copy (LSC), one small single copy (SSC), and two inverted repeats (IRs) [13]. It is an ideal tool for studying genetic differences and phylogenetic relationships among species due to its small length, high conservation, large number of gene copies and rapid evolution rate [14-16]. In recent years, with more and more chloroplast genomes being sequenced, the research on plant phylogeny based on chloroplast genome is increasing which provides an effective solution for systematic problems of some difficult taxa $[17,18]$.

At present, with the progress of genome sequencing technology and the increasing of genomics research, the taxonomic research of orchids has entered a stage of adjustment [19-23]. Therefore, the taxonomy system and phylogenetic status of orchids have been changed a lot, such as the Tulotis genus, which has been classified into the genus Platanthera in the present taxonomic studies since the morphological characteristics of this genus is very similar to that of Platanthera.

Platanthera ussuriensis (Regel) Maxim, formerly named as Tulotis ussuriensis, is a small terrestrial orchid species mainly distributed in China, Korean, Russia and Japan. Due to the increasing of habitat fragmentation and human disturbance, the survival of this species has been seriously threatened nowadays, and it has been listed as wild plant under State protection (category II) in China. In the present study, we sequenced the complete chloroplast genome of $P$. ussuriensis, and compared the resulting genome sequence with six other published chloroplast genomes of closed related species. Our aims were: (1) to investigate structural pattern of whole chloroplast genome including genome structure, gene order, and gene content; (2) to perform genome-based analysis and compare the differences among selected closely related species; (3) to reconstruct a chloroplast phylogeny for Platanthera orchids species using their whole chloroplast sequences. Our results would enrich the chloroplast genome database of Platanthera species, and get an insight into the evolutionary history of Orchidaceae family.

\section{Results}

\section{General feature of the chloroplast genome}

The chloroplast genome of $P$. ussuriensis is a circular molecule of 155,016 bp (Fig. 1), consisting of a large single copy (LSC) region of $83,984 \mathrm{bp}$, a small single copy (SSC) region of $17,936 \mathrm{bp}$, and a pair of inverted repeats (IRa and IRb) of $26,548 \mathrm{bp}$. The overall GC content was $36.7 \%$. The GC contents were unevenly distributed across different regions of the cp genome, which were found to be $34.2 \%, 29.3 \%$, and $43.1 \%$ for the LSC, SSC, and IR regions, respectively (Table 1 ). The chloroplast genome contained 132 genes, including 86 protein-coding genes, 38 tRNA genes and 8 rRNA genes. Among them, 92 genes were unique, and 20 genes were duplicated in the IR regions (4 rRNA genes, 8 tRNA genes and 8 proteincoding genes). There were 22 intron-containing genes including 14 protein-coding genes and 8 tRNA genes, and 3 genes $(r p s 12, y c f 3$ and $c l p P)$ possess two introns. In particular, $5^{\prime}$-end and $3^{\prime}$-end exons of rps 12 gene were respectively located in the LSC and IR regions (Table 2).

The amino acid frequency and codon usage were analyzed based on the 86 sequences of protein-coding genes which encoded a total of 26,587 codons. The results showed that leucine $(10.32 \%)$ was the most abundant amino acid, whereas cysteine $(1.21 \%)$ was the least abundance. The most and least used codons were AUU (1147) encoding lsoleucine, and UGC (78) encoding cysteine, respectively. Furthermore, the highest RSCU value was for UUA (1.94) in leucine, and the lowest was AGC (0.34) in serine and GAC (0.34) in aspartic acid, while methionine and tryptophan showed RSCU equals to 1 (Supplemental Table 1).

\section{Repeat and SSR analysis}

REPuter analysis revealed 38 oligonucleotide repeats (>30 bp) from which 16 were palindromic (P), 13 were forward (F), 5 were reverse (R), and 4 were complement (C). The repeat ranged from 30 to $39 \mathrm{bp}$ in length, and their repeat number varied from 1 to 4 . A total of 15 oligonucleotide repeats were identified in LSC region, 23 


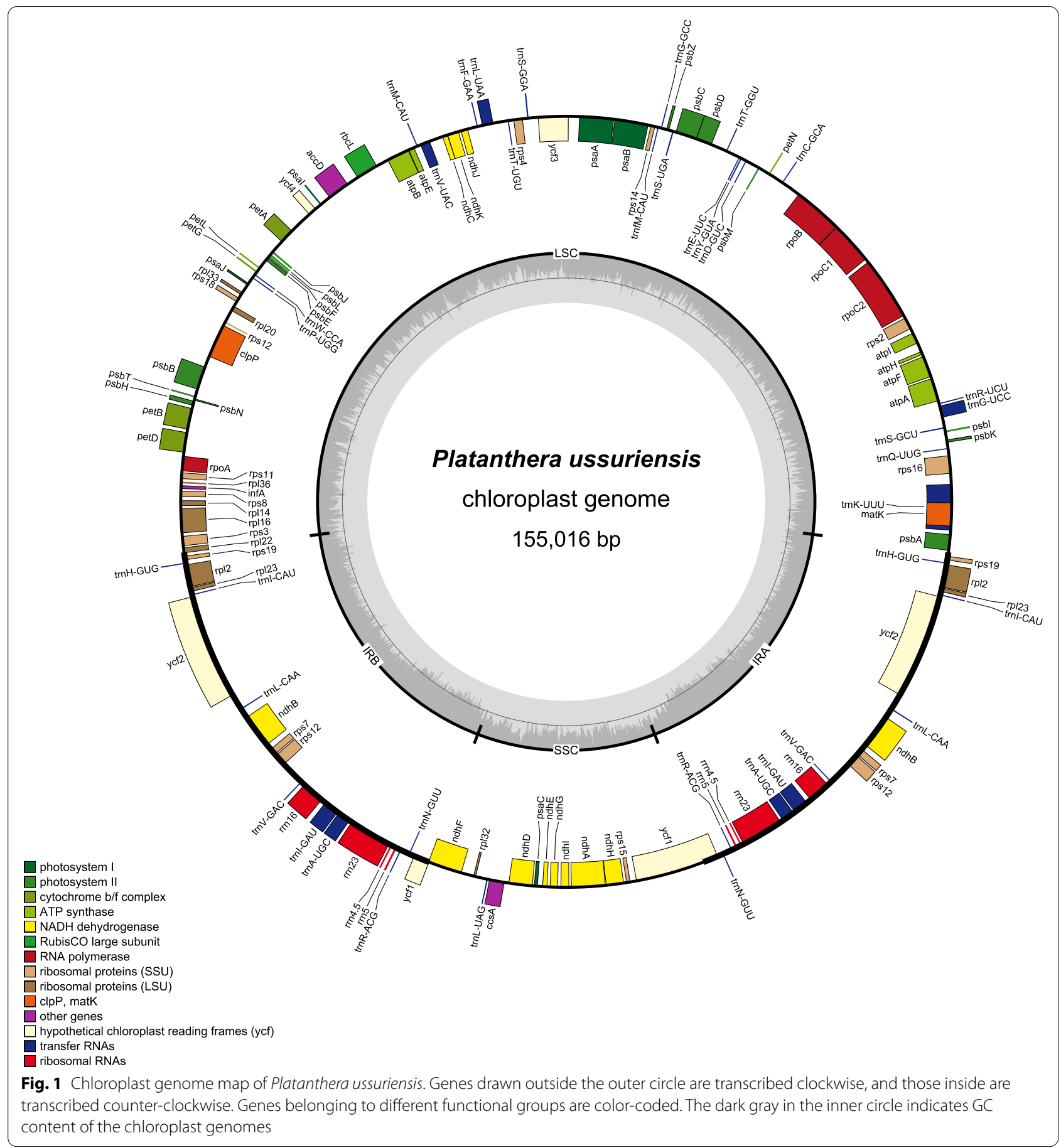

were distributed in junctions between the regions among which 21 were present in LSC/SSC region, and 2 were present in LSC/IR region (Supplemental Table 2, Fig. 2A). In addition, a total of 24 tandem repeats were identified (Supplemental Table 3). The length of these repeats varied from 7 to $29 \mathrm{bp}$, and their copy number ranged from 2 to 13 (Fig. 2B). Tandem repeats were distributed in all the
LSC, SSC and IRs regions, and 15, 6, and 3 were present in IGS, intron and CDS region, respectively (Fig. 2C).

A total of 104 SSRs were detected (Supplemental Table 4). Among these SSRs, there were 68, 23, 8 and 5 for mono-, di-, tri- and tetra- nucleotide repeats, respectively, and no pentanucleotide and hexanucleotide repeats were found (Fig. 3A). The majority of the 
Table 1 Comparative analysis of chloroplast genomes of four Platanthera species

\begin{tabular}{|c|c|c|c|c|c|}
\hline \multicolumn{2}{|c|}{ Characteristics } & Platanthera ussuriensis & Platanthera japonica & Platanthera chlorantha & $\begin{array}{l}\text { Platanthera } \\
\text { mandarinorum }\end{array}$ \\
\hline \multicolumn{2}{|c|}{ GenBank accession No. } & MN686021 & MN631092 & MK937914 & MN200370 \\
\hline \multicolumn{2}{|c|}{ Size (base pair, bp) } & 155,016 & 155,409 & 154,260 & 154,162 \\
\hline \multicolumn{2}{|c|}{ LSC length (bp) } & 83,984 & 84,049 & 83,279 & 83,325 \\
\hline \multicolumn{2}{|c|}{ SSC length (bp) } & 17,936 & 17,494 & 17,759 & 17,757 \\
\hline \multicolumn{2}{|l|}{ IR length (bp) } & 26,548 & 26,933 & 26,611 & 26,540 \\
\hline \multicolumn{2}{|c|}{ Number of genes } & 132 & 133 & 134 & 132 \\
\hline \multicolumn{2}{|c|}{ Protein-coding genes } & 86 & 87 & 88 & 86 \\
\hline \multicolumn{2}{|l|}{ tRNA genes } & 38 & 38 & 38 & 38 \\
\hline \multicolumn{2}{|l|}{ rRNA genes } & 8 & 8 & 8 & 8 \\
\hline \multirow[t]{4}{*}{ GC content } & Total (\%) & 36.7 & 36.9 & 36.7 & 36.9 \\
\hline & LSC (\%) & 34.2 & 34.4 & 34.3 & 34.3 \\
\hline & SSC (\%) & 29.3 & 29.7 & 29.4 & 29.5 \\
\hline & $\operatorname{IR}(\%)$ & 43.1 & 43.2 & 43.1 & 43.1 \\
\hline
\end{tabular}

mononucleotides were composed of $\mathrm{A} / \mathrm{T}$, and most of the dinucleotides were AT/AT (Fig. 3B). There were 76, 24, and 4 SSR repeats distributed in LSC, SSC, and IR region, respectively (Supplemental Table 5, Fig. 3C).

\section{Recognition of RNA editing sites}

PREP software found 35 genes predicting RNA editing sites in the chloroplast genome of $P$. ussuriensis among which 10 genes (petD, petG, petL, psaB, psbB, psbE, $p s b L, r p l 2, r p l 23, r p s 2)$ had been identified no sites (Supplemental Table 6). In the rest 25 protein-coding genes, a total of 72 RNA editing sites were predicted. Among these genes, the largest number of RNA editing sites were identified in the $n d h B$ gene (11 sites), following by $n d h D, n d h F, \operatorname{rpoB}$, and $r p o C 1$, with 6 sites respectively. All codon changes were $C$ to $T$ conversions, and 15 editing sites were the first nucleotide of the codon, while 57 editing sites were the second nucleotide of the codon. The most abundant amino acid change was $S$ to $L$, with 30 out of 72 editing events, and the rarest was $\mathrm{L}$ to $\mathrm{F}$, with only one out of all editing events (Fig. 4).

Table 2 Chloroplast gene content and functional classification in Platanthera ussuriensis

\begin{tabular}{|c|c|c|}
\hline Group of gene & Name of gene & Number \\
\hline Photosystem I & psaB, psaA, psal, psaJ, psaC & 5 \\
\hline Photosystem ॥ & $p s b A, p s b K, p s b l, p s b M, p s b D, p s b C, p s b Z, p s b J, p s b L, p s b F, p s b E, p s b B, p s b T, p s b N, p s b H$ & 15 \\
\hline Cytochrome b/f complex & petN, petA, petL, petG, petBa, petDa & 6 \\
\hline ATP synthase & $\operatorname{atp} A, \operatorname{atpF} F^{\mathrm{a}}, \operatorname{atpH}, \operatorname{atpl}, \operatorname{atp} E, \operatorname{atp} B$ & 6 \\
\hline NADH dehydrogenase & $n d h J, n d h K, n d h C, n d h B^{a}, c, n d h F, n d h D, n d h E, n d h G, n d h l, n d h A^{a}, n d h H$ & 12 \\
\hline RubisCO large subunit & $r b c L$ & 1 \\
\hline RNA polymerase & $r p o C 2, r p o C 1^{\mathrm{a}}, r p o B, r p o A$ & 4 \\
\hline Ribosomal proteins (SSU) & $\operatorname{rps} 12^{\mathrm{b}, \mathrm{c}}, r p s 16^{\mathrm{a}}, \operatorname{rps} 2, \operatorname{rps} 14, r p s 4, r p s 18, \operatorname{rps} 11, r p s 8, \operatorname{rps} 3, \operatorname{rps} 19^{\mathrm{c}}, \operatorname{rps} \mathcal{X}^{\mathrm{c}}, \operatorname{rps} 15$ & 15 \\
\hline Ribosomal proteins (LSU) & $r p / 33, r p / 20, r p / 36, r p / 14, r p / 16, r p / 22, r p / 2^{\mathrm{a}, \mathrm{c}}, r p / 23^{\mathrm{C}}, r p / 32$ & 11 \\
\hline Other genes & $a c c D$, infA, $\operatorname{ccs} A, c \mid p P^{b}$, matk & 5 \\
\hline $\begin{array}{l}\text { Hypothetical chloroplast reading frames } \\
\text { (ycf) }\end{array}$ & $y c f 3^{b}, y c f 4, y c f 2^{c}, y c f 7^{c}$ & 6 \\
\hline Transfer RNAs & 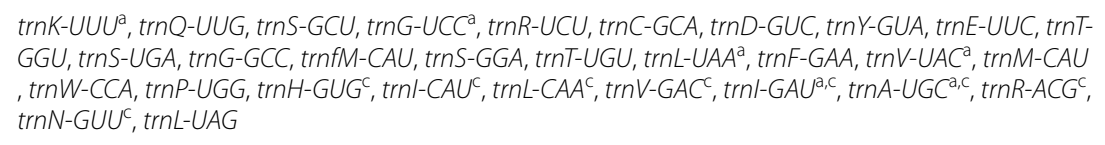 & 38 \\
\hline Ribosomal RNAs & $r r n 16^{c}, r r n 23^{c}, r r n 4.5^{c}, r r n 5^{c}$ & 8 \\
\hline Total & & 132 \\
\hline
\end{tabular}

${ }^{\mathrm{a}}$ Gene with one intron; ${ }^{\mathrm{b}} \mathrm{Gene}$ with two introns; ${ }^{\mathrm{c}} \mathrm{Gene}$ with two copies 

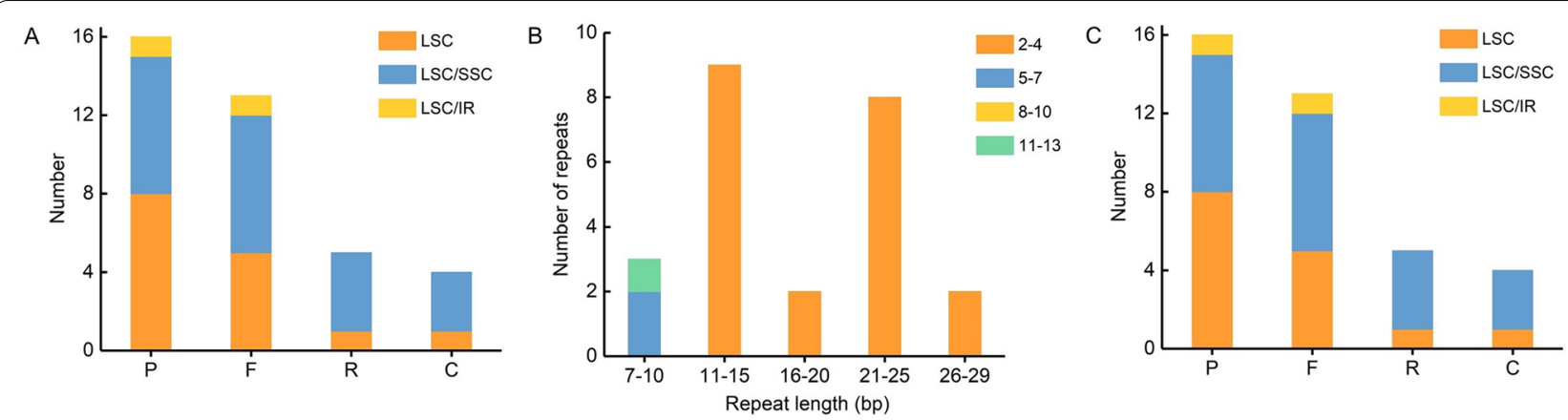

Fig. 2 Analysis of repeated sequences in the Platanthera ussuriensis chloroplast genome. A Frequency and locations of four repeat types, B Frequency of tandem repeats by length and their copy number, C Frequency of tandem repeats by their region and location

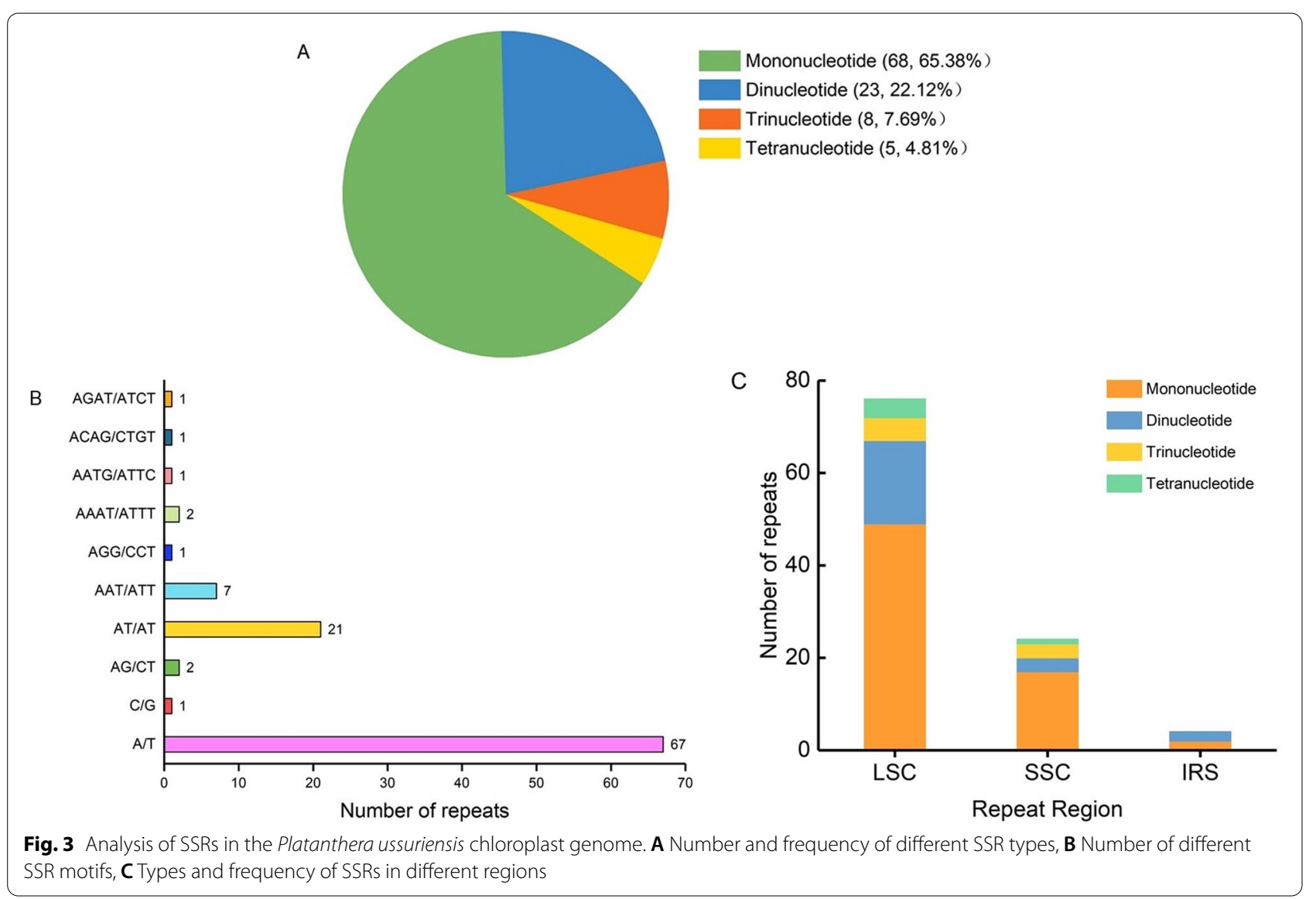

\section{IR expansion and contraction}

Comprehensive comparison at the LSC/IRs/SSC boundaries was analyzed among $P$. ussuriensis and six other closely related orchid species (Fig. 5). Although the length of the IR regions that ranged from $26,424 \mathrm{bp}$ to $26,933 \mathrm{bp}$, varied little among the seven species, some differences in the IR expansions and contractions were observed. These chloroplast genomes showed similar SSC/IR borders which were located in the coding region of the $n d h F(\mathrm{IRb} / \mathrm{SSC})$ and $y c f 1$ (SSC/IRa) gene, respectively. The IRb region extended into the rpl22 gene in all these chloroplast genomes, with the length ranging from 58 to $87 \mathrm{bp}$. The $r p l 22$ gene was duplicated only in Dactylorhiza majalis and $P$. chlorantha which located in the IRa/LSC border while not in the other five species. The $p s b A$ gene was included in the LSC region in all these species, 97-111 bp away from the IRa/LSC border. In addition, the $\operatorname{trn} H$ and rps 19 gene cluster were also 


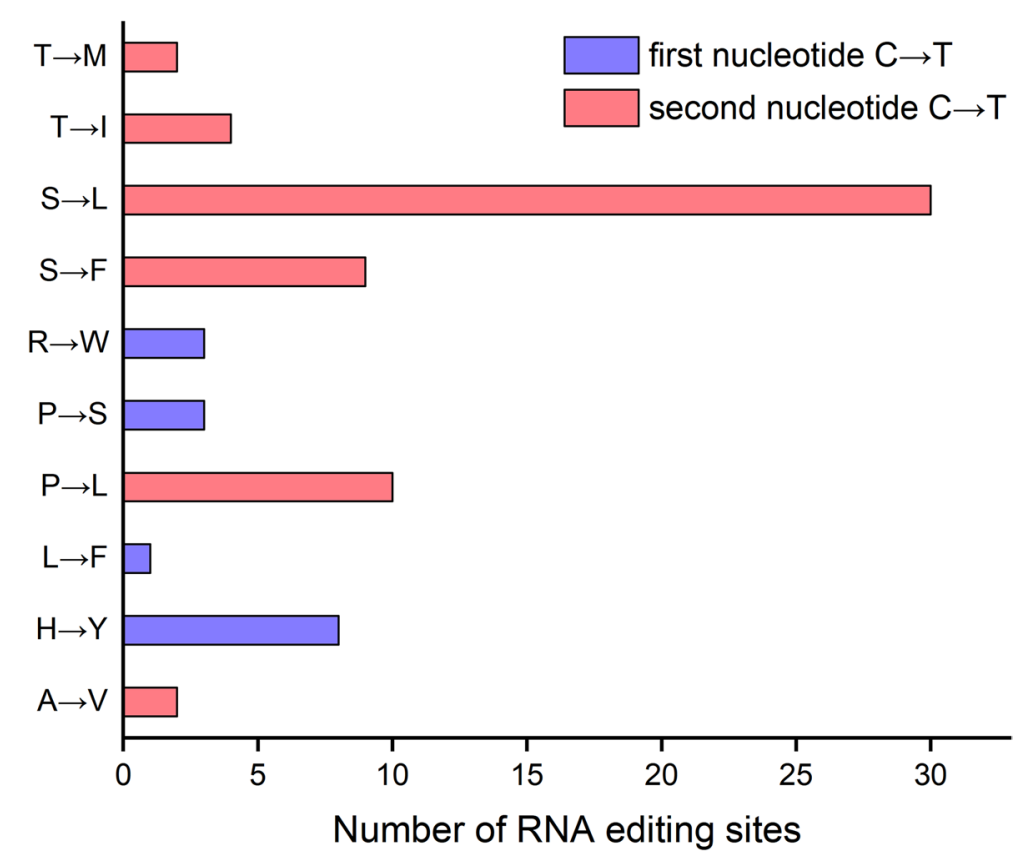

Fig. 4 Number of RNA editing sites by different amino acid changes and C to T location of the codons

totally located within the IR region and duplicated. The $y c f 1$ pseudogene was existed in all of these chloroplast genomes at the IRb/SSC border except for P. japonica, and an overlap was existed between $y c f 1$ pseudogene and $n d h F$ gene.

\section{Comparison of chloroplast genome sequences}

The chloroplast genome sequences were compared among $P$. ussuriensis and six other closely related orchid species (Fig. 6). Overall, the LSC and SSC regions in these chloroplast genomes were more divergent than the IR regions. Non-coding regions presented higher divergence than coding regions, but the rRNA genes of $P$. japonica had higher variability regions when comparing with the other six species. The highest polymorphic regions were located in the intergenic regions, such as trnK-UUU - matK, $n d h F-r p l 32$, and rpl32-trnL-UAG.

\section{Phylogenetic analysis}

The overall topology of the phylogenetic trees produced with Maximum-Likelihood (ML) and Neighbor-Joining (NJ) methods were similar based on our whole chloroplast genome sequences. The ML tree (Fig. 7) showed that all species formed five major clades which correspond to the five subfamilies of Orchidaceae. Within the Platanthera genus, all the Platanthera species were clustered in a single major clade, and $P$. ussuriensis and $P$. japonica were located in adjacent branches followed by the remaining Platanthera species.

\section{Discussion}

In this study, we reported the complete chloroplast genome of $P$. ussuriensis for the first time and compared with other six species of Orchidaceae in order to broaden the knowledge about the genome organization and molecular evolution of the Orchidaceae species.

The chloroplast genomes of angiosperms have conserved features and almost similar gene content and organization within and among different plant lineage $[24,25]$. The obtained chloroplast genome of P. ussuriensis in this study had typical characteristics of angiosperm chloroplast genomes, and the general feature were not significantly different from those of its closely related species $[10,26,27]$. However, the GC contents of these orchid species in IR region were remarkably higher than that in other two regions, and this phenomenon was also common in other plant species [28, 29]. The reason for this phenomenon is generally believed to be the existence of rRNA gene and tRNA gene within the region [30-32]. Although GC content of chloroplast genome varies greatly among different plant species $[16,17,20$, 22, 31-33], the GC content of different species of Platanthera genus in our study were very close.

Simple sequence repeats (SSRs), known as microsatellites, are very often used as genetic molecular markers in population genetic relationship and phylogenetic studies because of their widely distribution and highly polymorphism at the intraspecific level [15, 34-41]. In this study, more than 100 SSRs were found in the P. ussuriensis 


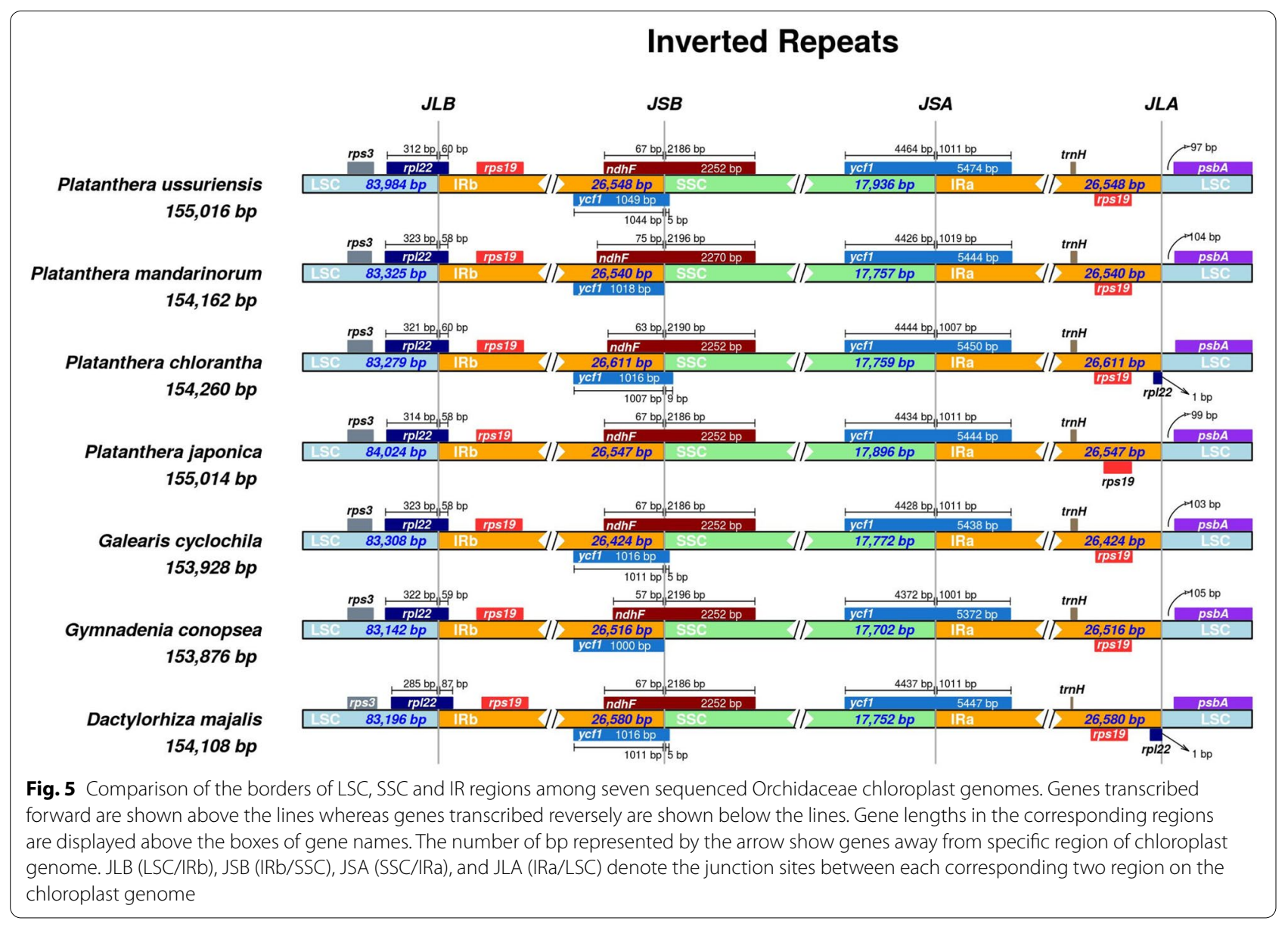

chloroplast genome. Most of them were AT rich, and the mononucleotides were found to be the most abundant repeats. Similar results have been found in other plant species, which were consistent with the contention that chloroplast SSRs are generally composed by short polyA or polyT repeats and the mononucleotide repeats tend to be the most frequent types of chloroplast SSRs [42, 43]. The oligonucleotide repeats found in this study could also be helpful for identification of polymorphic regions within P. ussuriensis and other Orchidaceae species.

RNA editing is one of the most important method to regulate gene expression in higher plants after transcription [44-46]. Of the 72 RNA editing sites of $P$. ussuriensis, $79.17 \%$ occurred at the second codons, and the rest occurred at the first codons. This phenomenon was consistent with the results of RNA editing in earlier studies $[47,48]$. It has been reported that most RNA editing tends to shift amino acids from polar to nonpolar and from hydrophilic to hydrophobic, which results in increased hydrophobicity of proteins [49-51]. In our present study, 61 of the 72 RNA editing sites were eventually converted to nonpolar and hydrophobic amino acids, including valine $(\mathrm{V})$, leucine $(\mathrm{L})$, isoleucine $(\mathrm{I})$, methionine (M), tryptophan (W) and phenylalanine (F). These results could provide data for the evolutionary investigations of the genus Platanthera, and the same was true for the analysis of codon usage frequency and relative synonymous codon usage (RSCU). In addition, codon usage was biased towards $\mathrm{A}$ and $\mathrm{U}$ at the third codon position, which is similar to the trend observed in most angiosperm chloroplast genomes [52].

Although the IR region of chloroplast genome is considered to be the most conservative region, the contraction and expansion of its borders are common events in the evolution of chloroplast genome, and therefore the main reason for the variation of chloroplast genome length [53-55]. In eudicots, the trnH gene is usually located in the LSC region and the borders of the IR regions are found to contain the rps19 or rps19 pseudogene [56-59], while IRb/LSC is located downstream of the $p s b A$ gene and IRs have expanded to include $\operatorname{trnH}$ and rps19 gene cluster in most monocots [60, 61]. However, the study of IR junctions between LSC and SSC showed remarkable changes. Our results showed that the 


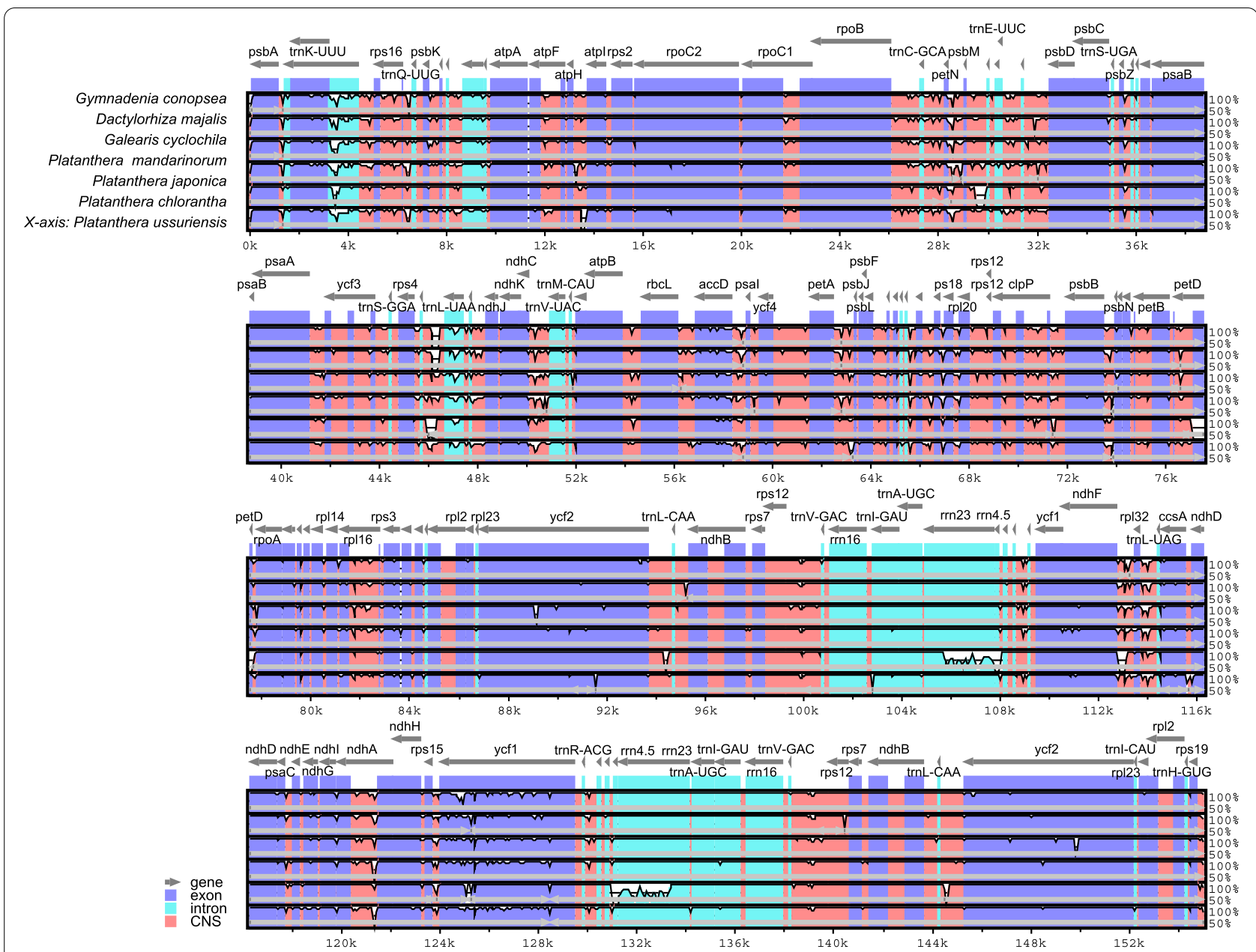

Fig. 6 Sequence alignment of seven Orchidaceae chloroplast genomes using mVISTA. The vertical scale indicates the percentage of identity, ranging from 50 to 100\%. The horizontal axis indicated the coordinates within the chloroplast genome. Genome regions are color coded as exton, intron, and conserved non-coding sequences (CNS)

trnH and rps19 gene cluster was included in the IR region of the orchid species chloroplast genome, which was consistent with its location in most monocot genomes. However, the IR expanded into the $r p l 22$ gene in all of these orchid species and created a pseudogene at the IRa/LSC border in D. majalis and P. chlorantha species. Similar results were also found in other orchid species [62] while was significantly different from that in other monocot lineages [24, 61]. The fluxes in the IR boundaries have been suggested to implicate the taxonomic relationships among angiosperms, and our results revealed that the expansion was unusual in Orchidaceae species and the degrees of IR to LSC expansions were much larger than other monocot lineages. Variation of expansions has also been detected at IR/SSC boundaries of monocot chloroplast genomes, and there was an IR contraction existed in Phalaenopsis chloroplast genome in which the $y c f l$ gene was completely included in SSC region [62]. In our study, the $y c f 1$ gene spanned the SSC/IRa boundary in Platanthera and adjacent species, indicating that the degrees of expansion may varied greatly among different orchid genus. These differences would be useful for studying the taxonomy and evolutionary relationships of different species.

$\mathrm{ML}$ and NJ analysis were used to construct the evolutionary tree, and we found that ML and NJ trees had similar phylogenetic topologies with five major clades (Orchidoideae, Epidendroideae, Cypripedioideae, Vanilloideae, and Apostasioideae). The Platanthera branch had a strong supported topology and showed that $P$. ussuriensis and P. japonica have a closer evolutionary relationship. This phylogenetic topology was highly consistent with previously published phylogenetic analysis based on complete plastid genomes [10] and study based on several genes from Orchidaceae [63]. The data we presented here and in conjunction with previously published 


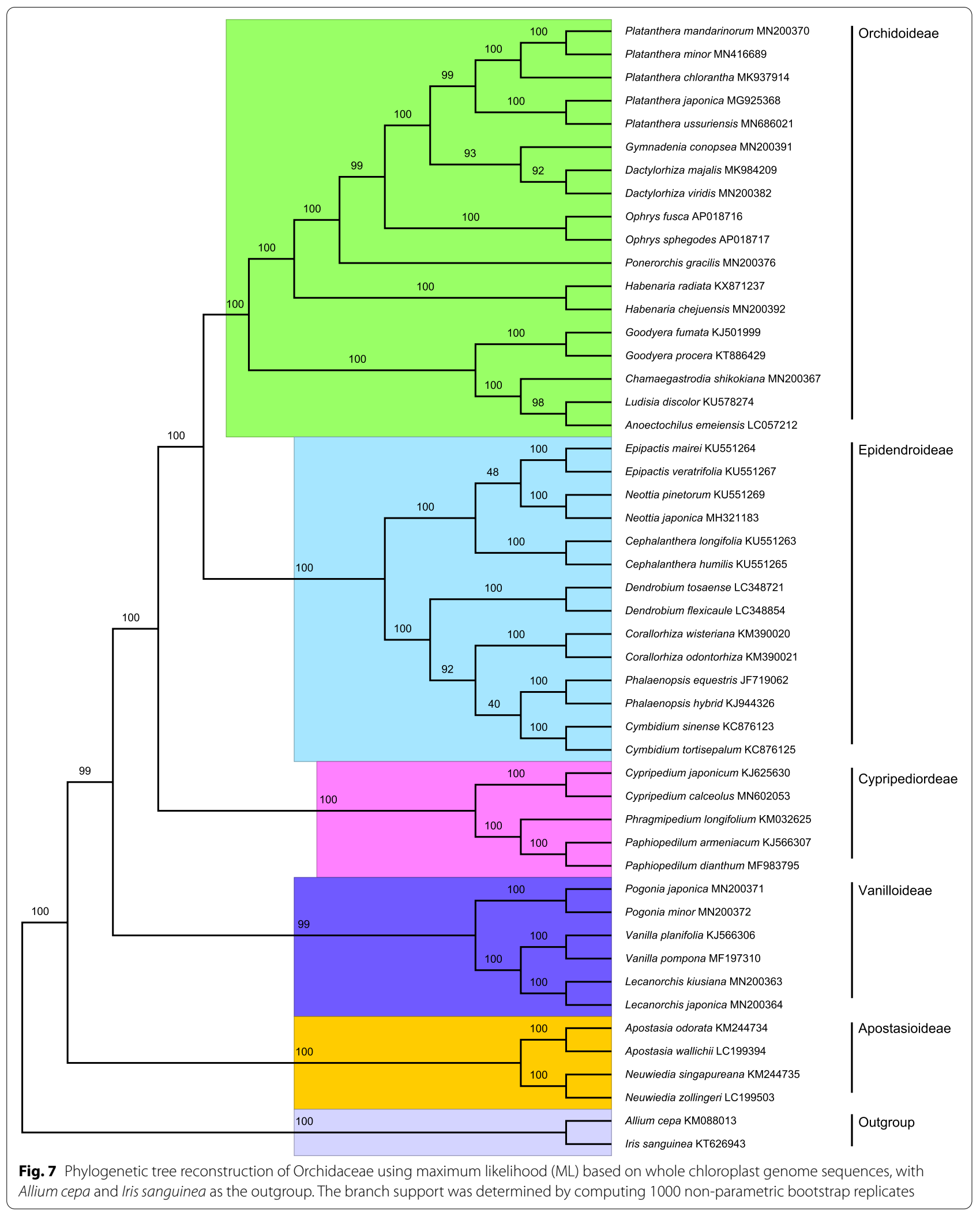


chloroplast genome sequences would help to expand our understanding of the evolutionary history of the orchid species, especially for the systematics and evolution of the Platanthera genus.

\section{Conclusion}

Platanthera ussuriensis, formerly named as Tulotis ussuriensis, is a small terrestrial orchid species and has been listed as wild plant under State protection (category II) in China. A great understanding of genomic information provides insights into the conservation works of Platanthera species. This research focused on the chloroplast sequence analysis of $P$. ussuriensis species, including general feature, SSR sites, RNA editing sites, and comparison with other closely related species. The results provide a guide for future genomic and conservation strategy of the Orchidaceae species.

\section{Methods}

\section{Ethical statement}

No specific permits were required for the collection of specimens for this study. This research was carried out in compliance with the relevant laws of China.

\section{Plant materials and chloroplast genome sequencing}

Fresh leaves from an adult $P$. ussuriensis plant were collected and identified by Dr. Bo Qu and Dr. Xuhui Chen of Shenyang Agricultural University from Daqinggou nature reserve, Fuxin, northeast China. Total genomic DNA was extracted using the CTAB method [64], and the specimen was stored with the archival number of ORCHID_PLA_USS_01 at College of Bioscience and Biotechnology of Shenyang Agricultural University. Nextgeneration sequencing was performed with an Illumina Miseq sequencing platform by Shanghai Personal Biotechnology Co. Ltd, China. The paired-end library was constructed using TruSeqTM DNA Sample Prep Kit, and then paired reads with 400 bp insert size were sequenced and yielded at least 2 GB clean data. The qualities of the clean reads were checked using FastQC v0.11.7.

\section{Chloroplast genome assembly and annotation}

For de novo chloroplast genome assembly, all the pairedend sequences were assembled into contigs, and neighboring contigs with paired-end support for continuity were merged into scaffolds using SPAdes v.3.11.0 software. Then, using the published chloroplast genome and protein coding gene sequences of related species as reference, the scaffolds with gene matching were picked out and assembled into the chloroplast genome with Velvet v.1.2.07 [65]. The online GeSeq-Annotation of Organellar Genomes program coupled with manual corrections for start and stop codons were used to annotate the complete chloroplast genome [66]. The tRNA genes were identified using GeSeq and tRNAscan-SE v2.0.5 [67]. A circular genome map was drawn using the OGDRAW program (https://chlorobox.mpimp-golm.mpg.de/OGDraw) [68].

\section{Sequence analysis and statistics}

The web-based software REPuter (https://bibiserv.cebit ec.uni-bielefeld.de/reputer/) [69] was used to analyze the repeat sequences, which included forward, reverse, complement and palindromic repeats with minimal repeat size set to $30 \mathrm{bp}$, hamming distance set to 3 and maximum computed repeats set to 90 . In addition, the tandem repeat sequences were detected by Tandem Repeats Finder with default parameters (http://tandem.bu.edu/ trf/trf.html) [70]. Simple sequence repeats(SSR) which is a tract of repetitive DNA and usually ranges in length from 1 to 6 nucleotides were detected via MISA (https:// webblast.ipk-gatersleben.de/misa/) by setting the minimum number of repeats to 10, 5, 4, 3, 3 and 3 for mononucleotide, dinucleotide, trinucleotide, tetranucleotide, pentanucleotide and hexanucleotide, respectively [71].

Condon usage was analyzed by MEGA-X software (version 10.1.6) [72], and the relative synonymous codon usage (RSCU) and amino acid frequencies were calculated with default settings [73]. The putative RNA editing sites of 86 protein-coding genes in P. ussuriensis were predicted by the online PREP-Cp program (http://prep. unl.edu/) with default parameters, and the cutoff value was set to 0.8 to ensure prediction accuracy [74].

\section{Sequence divergence and genome comparison}

The mVISTA program in LAGAN mode [75] was used to make pairwise alignments and sequence divergence within $P$. ussuriensis and six other closely related species, i.e., P. chlorantha, P. japonica, P. mandarinorum, Galearis cyclochila, D. majalis and Gymnadenia conopsea. The result was plotted using the mVISTA tools (http:// genome.lbl.gov/vista/mvista/submit.shtml) with $P$. ussuriensis as a reference [76]. The contraction and expansion of the IR boundaries between the four main regions (LSC/IRa/SSC/IRb) of the seven chloroplast genome sequences were visualized using the online software IRSCOPE (https://irscope.shinyapps.io/irapp/) [77].

\section{Phylogenetic analysis}

To determine the location of P. ussuriensis in Orchidaceae family and to analyze the phylogenetic relationship of the Platanthera genus, a total of 47 chloroplast genomes from the family Orchidaceae were selected to construct phylogenetic trees, with two species Allium cepa and Iris sanguinea selected as the outgroup. We downloaded chloroplast genome sequences of 46 species across Orchidaceae family which correspond to 
all five subfamilies of Orchidaceae (Orchidoideae, Epidendroideae, Cypripediordeae, Vanilloideae, and Apostasioideae), selecting two chloroplast genomes per genus except where there was only one representative species in any genus which was also included in the analyses. These sequences were aligned using HomBlocks [78] with the Gblocks method [79], resulting in 9,262 aligned characters. Maximum-Likelihood (ML) and Neighbor-Joining (NJ) analyses were performed. ML tree was built using PhyML 3.0 [80] with a General Time Reversible + Proportion Invariant + Gamma $(\mathrm{GTR}+\mathrm{I}+\mathrm{G})$ model. NJ tree was bulit under Maximum Composite Likelihood method with Gamma distribution rates (parameter $=4$ ) using MEGA6 [81]. 1000 non-parametric bootstrap replicates were performed to estimate the support of the data for each internal branch of the phylogeny.

\author{
Abbreviations \\ C: Complement; CDS: Coding sequence; CNS: Conserved non-coding \\ sequences; F: Forward; IGS: Intergenic spacer; IRs: Inverted repeats; LSC: Large \\ single copy; ML: Maximum-Likelihood; NJ: Neighbor-Joining; P: Palindromic; R: \\ Reverse; RSCU: Relative synonymous codon usage; SSC: Small single copy; SSR: \\ Simple sequence repeat.
}

\section{Supplementary Information}

The online version contains supplementary material available at https://doi. org/10.1186/s12864-022-08319-9.

Additional file 1.

Acknowledgements

We are grateful to Mr Lei Wu for his help with the experimental software.

\section{Authors' contributions}

$\mathrm{CH}, \mathrm{RD}, \mathrm{XZ}$, and $\mathrm{LZ}$ collaborated on the analysis and writing of this manuscript. $X C$ contributed on the design and editing of this manuscript. BQ provided the plant material. All authors reviewed and approved the final manuscript.

\section{Funding}

This work was carried out with the financial support of Special Project of Orchid Survey of National Forestry and Grassland Administration (grant number 2021070710), China Agriculture Research System of MOF and MARA (grant number CARS-23-B17), the Foundation of Liaoning "Xingliaoyingcai Plan" (grant number XLYC1807180); and the National Natural Science Foundation of China (grant number 31670378).

\section{Availability of data and materials}

The datasets generated or analyzed during the current study are available in the NCBI Bioproject repository MN686021, (https://www.ncbi.nlm.nih.gov/ nuccore/MN686021.1)

\section{Declarations}

\section{Ethics approval and consent to participate}

Since the Special Project of Orchid Survey of National Forestry and Grassland Administration, Dr. Xuhui Chen has obtained the permission by Shenyang Agricultural University to collect plant species from Daqinggou nature reserve, Fuxin, northeast China. Because of the important protective value of Platanthera ussuriensis, Dr. Chen only collected a few leaves from the plants for further molecular study without damaging the plant growth. The plant material collection and experimental research were conducted according to the Plant Protection and Regulation of Shenyang Agricultural University.

\section{Consent for publication}

Not applicable

\section{Competing interests}

The authors declare that they have no competing interests.

\section{Author details}

${ }^{1}$ College of Bioscience and Biotechnology, Shenyang Agricultural University, Shenyang 110161, China. ${ }^{2}$ College of Land and Environment, Shenyang Agricultural University, Shenyang 110161 , China. ${ }^{3}$ College of Forestry, Shenyang Agricultural University, Shenyang 110161, China.

Received: 1 July 2021 Accepted: 17 January 2022

Published online: 27 January 2022

\section{References}

1. Christenhusz MJM, Byng JW. The number of known plants species in the world and its annual increase. Phytotaxa. 2016;261(3):201-17.

2. Chase MW, Cameron KM, Freudenstein JV, Pridgeon AM, Schuiteman A. An updated classification of Orchidaceae. Bot J Linn Soc. 2015;177(2):151-74

3. Givnish TJ, Spalink D, Ames M, Lyon SP, Hunter SJ, Zuluaga A, lles WJD, Clements MA, Arroyo MTK, Leebens-Mack J, et al. Orchid phylogenomics and multiple drivers of their extraordinary diversification. P Roy Soc B-Biol Sci. 1814;2015(282):171-80

4. Xu X, Ding C-C, Hu W, Yu X, Zheng Y, Zhang D, Liao X, Liu X, Liu Z-J, Lan S. Cymbidium xichouense (Orchidaceae; Epidendroideae), a new species from China: evidence from morphological and molecular data. Phytotaxa. 2021:484(3):291-7.

5. Li H, Xiao W, Tong T, Li Y, Zhang M, Lin X, Zou X, Wu Q, Guo X. The specific DNA barcodes based on chloroplast genes for species identification of Orchidaceae plants. Sci Rep. 2021;11(1):1424.

6. Kim HM, Oh SH, Bhandari GS, Kim CS, Park CW. DNA barcoding of Orchidaceae in Korea. Mol Ecol Resour. 2014;14(3):499-507.

7. Feng $S$, Jiang Y, Wang S, Jiang M, Zhe C, Ying Q, Wang H. Molecular identification of Dendrobium species (Orchidaceae) based on the DNA barcode ITS2 region and its application for phylogenetic study. Int J Mol Sci. 2015;16(9):21975-88.

8. Techen N, Parveen I, Pan Z, Khan IA. DNA barcoding of medicinal plant material for identification. Curr Opin Biotechnol. 2014:25:103-10.

9. Raskoti BB, Jin WT, Xiang XG, Schuiteman A, Li DZ, Li JW, Huang WC, Jin $\mathrm{XH}$, Huang LQ. A phylogenetic analysis of molecular and morphological characters of Herminium (Orchidaceae, Orchideae): evolutionary relationships, taxonomy, and patterns of character evolution. Cladistics. 2016;32(2):198-210.

10. Kim YK, Jo S, Cheon SH, Joo MJ, Hong JR, Kwak M, Kim KJ. Plastome evolution and phylogeny of Orchidaceae, with 24 new sequences. Front Plant Sci. 2020;11:322.

11. Li MH, Zhang GQ, Lan SR, Liu ZJ. China Phylogeny Consortium. A molecular phylogeny of Chinese orchids. J Syst Evol. 2016;54(4):349-62.

12. Neuhaus HE, Emes MJ. Nonphotosynthetic metabolism in plastids. Annu Rev Plant Physiol Plant Mol Biol. 2000;51:111-40.

13. Daniell H, Lin CS, Yu M, Chang WJ. Chloroplast genomes: diversity, evolution, and applications in genetic engineering. Genome Biol. 2016;17(1):134.

14. Thomson RC, Wang IJ, Johnson JR. Genome-enabled development of DNA markers for ecology, evolution and conservation. Mol Ecol. 2010;19(11):2184-95.

15. Dong WP, Xu C, Cheng T, Lin K, Zhou SL. Sequencing angiosperm plastid genomes made easy: a complete set of universal primers and a case study on the phylogeny of Saxifragales. Genome Biol Evol. 2013;5(5):989-97.

16. Liu LX, Li R, Worth JRP, Li X, Li P, Cameron KM, Fu CX. The complete chloroplast genome of Chinese Bayberry (Morella rubra, Myricaceae): 
implications for understanding the evolution of Fagales. Front Plant Sci. 2017;8:968.

17. de Abreu NL, Alves RJV, Cardoso SRS, Bertrand YJK, Sousa F, Hall CF, Pfeil $\mathrm{BE}$, Antonelli A. The use of chloroplast genome sequences to solve phylogenetic incongruences in Polystachya Hook (Orchidaceae Juss). PeerJ. 2018;6(1):e4916.

18. Zhang R, Wang YH, Jin JJ, Stull GW, Bruneau A, Cardoso D, De Queiroz LP, Moore MJ, Zhang SD, Chen SY, et al. Exploration of plastid phylogenomic conflict yields new insights into the deep relationships of Leguminosae. Syst Biol. 2020;69(4):613-22.

19. Li ZH, Jiang Y, Ma X, Li JW, Yang JB, Wu JY, Jin XH. Plastid genome evolution in the subtribe Calypsoinae (Epidendroideae, Orchidaceae). Genome Biol Evol. 2020;12(6):867-70

20. Li YX, Li ZH, Schuiteman A, Chase MW, Li JW, Huang WC, Hidayat A, Wu SS, Jin XH. Phylogenomics of Orchidaceae based on plastid and mitochondrial genomes. Mol Phylogenet Evol. 2019;139:106540.

21. Li ZH, Ma X, Wang DY, Li YX, Wang CW, Jin XH. Evolution of plastid genomes of Holcoglossum (Orchidaceae) with recent radiation. BMC Evol Biol. 2019;19(1):63.

22. Wei Z, Chen B, Cao Y, Zheng Y, Zhang Y, Zhao K, Zhou Y. The complete chloroplast genome of Cymbidium hookerianum (Orchidaceae): genome structure and basic analysis. Mitochondrial DNA B. 2021;6(1):36-7.

23. Zavala-Paez M, Vieira LDN, de Baura VA, Balsanelli E, de Souza EM, Cevallos MC, Chase MW, Smidt EC. Comparative plastid genomics of neotropical Bulbophyllum (Orchidaceae; Epidendroideae). Front Plant Sci. 2020;11:799.

24 Yang $M$, Zhang XW, Liu GM, Yin YX, Chen KF, Yun QZ, Zhao DJ, AlMssallem IS, Yu J. The complete chloroplast genome sequence of Date (Phoenix dactylifera L.). Palm PLoS One. 2010;5(9):e12762.

25. Lin H, Sun YN, Lan SR, Ma XK. The complete plastome of Cymbidium tortisepalum (Orchidaceae) hermaphrodite. Mitochondrial DNA B. 2020;5(2):1265-6.

26. Zhang $X$, Li HZ, Huang H, Wang QL. The complete plastome sequence of Platanthera japonica (Orchidaceae): an endangered medicinal and ornamental plant. Mitochondrial DNA B. 2020;5(1):468-9.

27. Lallemand F, May M, Ihnatowicz A, Jakalski M. The complete chloroplast genome sequence of Platanthera chlorantha (Orchidaceae). Mitochondrial DNA B. 2019;4(2):2683-4.

28. Shen XF, Wu ML, Liao BS, Liu ZX, Bai R, Xiao SM, Li XW, Zhang BL, Xu $J$, Chen SL. Complete chloroplast genome sequence and phylogenetic analysis of the medicinal plant Artemisia annua. Molecules. 2017;22(8):1330.

29. Guo S, Guo LL, Zhao W, Xu J, Li YY, Zhang XY, Shen XF, Wu ML, Hou XG. Complete chloroplast genome sequence and phylogenetic analysis of Paeonia ostii. Molecules. 2018;23(2):246.

30. Liu HY, Yu Y, Deng YQ, Li J, Huang ZX, Zhou SD. The chloroplast genome of Lilium henrici: genome structure and comparative analysis. Molecules. 2018;23(6):1276

31. Zhou JG, Cui YX, Chen XL, Li Y, Xu ZC, Duan BZ, Li YH, Song JY, Yao H. Complete chloroplast genomes of Papaver rhoeas and Papaver orientale: molecular structures, comparative analysis, and phylogenetic analysis. Molecules. 2018;23(2):437.

32. Meng J, Li XP, Li HT, Yang JB, Wang H, He J. Comparative analysis of the complete chloroplast genomes of four Aconitum medicinal species. Molecules. 2018;23(5):1015.

33. Wicke S, Müller KF, de Pamphilis CW, Quandt D, Wickett NJ, Zhang Y, Renner SS, Schneeweiss GM. Mechanisms of functional and physical genome reduction in photosynthetic and nonphotosynthetic parasitic plants of the Broomrape family. Plant Cell. 2013;25:3711-25.

34. Dong WP, Xu C, Li DL, Jin XB, Li RL, Lu Q, Suo ZL. Comparative analysis of the complete chloroplast genome sequences in psammophytic Haloxylon species (Amaranthaceae). PeerJ. 2016;4(2):e2699.

35. Saina JK, Li Z-Z, Mekbib Y, Gichira AW, Liao Y-Y. Transcriptome sequencing and microsatellite marker discovery in Ailanthus altissima (Mill.) Swingle (Simaroubaceae). Mol Biol Rep. 2021;48:2007-23.

36. Yang YC, Zhou T, Duan D, Yang J, Feng L, Zhao GF. Comparative analysis of the complete chloroplast genomes of five Quercus species. Front Plant Sci. 2016;7:959.
37. Provan J, Corbett G, McNicol JW, Powell W. Chloroplast DNA variability in wild and cultivated rice (Oryza spp.) revealed by polymorphic chloroplast simple sequence repeats. Genome. 1997;40(1):104-10.

38 Angioi SA, Desiderio F, Rau D, Bitocchi E, Attene G, Papa R. Development and use of chloroplast microsatellites in Phaseolus spp. and other legumes. Plant Biology. 2009;11(4):598-612.

39. Ebert $D$, Peakall R. A new set of universal de novo sequencing primers for extensive coverage of noncoding chloroplast DNA: new opportunities for phylogenetic studies and cpSSR discovery. Mol Ecol Resour. 2009;9(3):777-83.

40. Wheeler GL, Dorman HE, Buchanan A, Challagundla L, Wallace LE. A review of the prevalence, utility, and caveats of using chloroplast simple sequence repeats for studies of plant biology. Appl Plant Sci. 2014:2(12):1400059.

41. Cheng Y, de Vicente MC, Meng H, Guo W, Tao N, Deng X. A set of primers for analyzing chloroplast DNA diversity in Citrus and related genera. Tree Physiol. 2005;25(6):661-72.

42. Provan J, Powell W, Hollingsworth PM. Chloroplast microsatellites: new tools for studies in plant ecology and evolution. Trends Ecol Evol. 2001;6(3):142-7.

43. Kuang DY, Wu H, Wang YL, Gao LM, Zhang SZ, Lu L. Complete chloroplast genome sequence of Magnolia kwangsiensis (Magnoliaceae): implication for DNA barcoding and population genetics. Genome. 2011;54(8):663-73.

44. Brennicke A, Marchfelder A, Binder S. RNA editing. FEMS Microbiol Rev. 1999:23(3):297-316.

45. Bock R. Sense from nonsense: How the genetic information of chloroplasts is altered by RNA editing. Biochimie. 2000;82(6-7):549-57.

46. Kumbhar F, Nie X, Xing G, Zhao X, Weining S. Identification and characterisation of RNA editing sites in chloroplast transcripts of einkorn wheat (Triticum monococcum): RNA editing sites in einkorn wheat plastome. Ann Appl Biol. 2018;172(2):197-207.

47 Park M, Park H, Lee H, Lee BH, Lee J. The complete plastome sequence of an Antarctic bryophyte Sanionia uncinata (Hedw.) Loeske. Int J Mol Sci. 2018;19(3):709.

48. Chen H, Deng L, Jiang Y, Lu P, Yu J. RNA editing sites exist in protein-coding genes in the chloroplast genome of Cycas taitungensis. J Integr Plant Biol. 2011;53(12):961-70.

49. Wang W, Yu H, Wang J, Lei W, Gao J, Qiu X, Wang J. The complete chloroplast genome sequences of the medicinal plant Forsythia suspensa (Oleaceae). Int J Mol Sci. 2017;18(11):2288.

50. Zhou J, Chen X, Cui Y, Sun W, Li Y, Wang Y, Song J, Yao H. Molecular structure and phylogenetic analyses of complete chloroplast genomes of two Aristolochia medicinal species. Int J Mol Sci. 2017;18(9):1839.

51. de Santana Lopes A, Gomes Pacheco T, Nimz T, do Nascimento Vieira L, Guerra MP, Nodari RO, de Souza EM, de Oliveira Pedrosa F, Rogalski M. The complete plastome of macaw palm [Acrocomia aculeata (Jacq.) Lodd. ex Mart.] and extensive molecular analyses of the evolution of plastid genes in Arecaceae. Planta. 2018;247(4):1011-30.

52. Liu Q, Xue Q. Comparative studies on codon usage pattern of chloroplasts and their host nuclear genes in four plant species. J Genet. 2005:84(1):55-62.

53. Kode V, Mudd EA, lamtham S, Day A. The tobacco plastid accD gene is essential and is required for leaf development. Plant J. 2010:44(2):237-44.

54. Raubeson LA, Peery R, Chumley TW, Dziubek C, Fourcade HM, Boore $\mathrm{JL}$, Jansen RK. Comparative chloroplast genomics: analyses including new sequences from the angiosperms Nuphar advena and Ranunculus macranthus. BMC Genom. 2007:8:174.

55. Yao X, Tang P, Li Z, Li D, Liu Y, Huang H. The first complete chloroplast genome sequences in Actinidiaceae: genome structure and comparative analysis. PLoS One. 2015;10(6):e0129347.

56. Ma Q, Li S, Bi C, Hao Z, Sun C, Ye N. Complete chloroplast genome sequence of a major economic species, Ziziphus jujuba (Rhamnaceae). Curr Genet. 2017;63(1):117-29.

57. Wang S, Yang C, Zhao X, Chen S, Qu GZ. Complete chloroplast genome sequence of Betula platyphylla: gene organization, RNA editing, and comparative and phylogenetic analyses. BMC Genom. 2018;19(1):950.

58. Mehmood F, Abdullah, Shahzadi I, Ahmed I, Waheed MT, Mirza B. Characterization of Withania somnifera chloroplast genome and its comparison with other selected species of Solanaceae. Genomics. 2020;112(2):1522-30. 
59. Zhang RS, Yang J, Hu HL, Xia RX, Li YP, Su JF, Li Q, Liu YQ, Qin L. A high level of chloroplast genome sequence variability in the Sawtooth Oak Quercus acutissima. Int J Biol Macromol. 2020;152:340-8.

60. Wang RJ, Cheng CL, Chang CC, Wu CL, Su TM, Chaw SM. Dynamics and evolution of the inverted repeat-large single copy junctions in the chloroplast genomes of monocots. BMC Evol Biol. 2008;8:36.

61. Huotari T, Korpelainen H. Complete chloroplast genome sequence of Elodea canadensis and comparative analyses with other monocot plastid genomes. Gene. 2012;508(1):96-105.

62. Chang CC, Lin HC, Lin IP, Chow TY, Chen HH, Chen WH, Cheng CH, Lin CY, Liu SM, Chang CC, et al. The chloroplast genome of Phalaenopsis aphrodite (Orchidaceae): comparative analysis of evolutionary rate with that of grasses and its phylogenetic implications. Mol Biol Evol. 2006;23(2):279-91.

63. Jin WT, Schuiteman A, Chase MW, Li JW, Chung SW, Hsu TC, Jin XH. Phylogenetics of subtribe Orchidinae s.l. (Orchidaceae; Orchidoideae) based on seven markers (plastid matK, psaB, rbcL, trnL-F, trnH-psbA, and nuclear nrlTS, Xdh): implications for generic delimitation. BMC Plant Biol. 2017;17(1):222.

64. Doyle JJ. A rapid DNA isolation procedure for small quantities of fresh leaf tissue. Phytochem Bull. 1987;19:11-5.

65. Zerbino DR, Birney E. Velvet: algorithms for de novo short read assembly using de Bruijn graphs. Genome Res. 2008;18(5):821-9.

66. Michael T, Pascal L, Tommaso P, Ulbricht-Jones ES, Axel F, Ralph B, Stephan G. GeSeq-versatile and accurate annotation of organelle genomes. Nucleic Acids Res. 2017;W1:W6-11.

67. Lowe TM, Chan PP. tRNAscan-SE On-line: integrating search and context for analysis of transfer RNA genes. Nucleic Acids Res. 2016;44(W1):W54-7.

68. Lohse M, Drechsel O, Bock R. OrganellarGenomeDRAW (OGDRAW): a tool for the easy generation of high-quality custom graphical maps of plastid and mitochondrial genomes. Curr Genet. 2007;52(5-6):267-74.

69. Kurtz S, Choudhuri JV, Ohlebusch E, Schleiermacher C, Stoye J, Giegerich R. REPuter: the manifold applications of repeat analysis on a genomic scale. Nucleic Acids Res. 2001;29(22):4633-42.

70. Benson G. Tandem repeats finder: a program to analyze DNA sequences. Nucleic Acids Res. 1999;27(2):573-80.

71. Beier S, Thiel T, Munch T, Scholz U, Mascher M. MISA-web: a web server for microsatellite prediction. Bioinformatics. 2017;33(16):2583-5.

72. Kumar S, Stecher G, Li M, Knyaz C, Tamura K. MEGA X: molecular evolutionary genetics analysis across computing platforms. Mol Biol Evol. 2018;35(6):1547-9.

73 Wolfe KH, Li WH, Sharp PM. Rates of nucleotide substitution vary greatly among plant mitochondrial, chloroplast, and nuclear DNAs. PNAS. 1987;84(24):9054-8.

74. Mower JP. The PREP suite: predictive RNA editors for plant mitochondrial genes, chloroplast genes and user-defined alignments. Nucleic Acids Res. 2009;37:W253-9.

75 Frazer KA, Pachter L, Poliakov A, Rubin EM, Dubchak I. VISTA: computational tools for comparative genomics. Nucleic Acids Res. 2004:32:W273-279.

76. Mayor C, Brudno M, Schwartz JR, Poliakov A, Rubin EM, Frazer KA, Pachter LS, Dubchak I. VISTA : visualizing global DNA sequence alignments of arbitrary length. Bioinformatics. 2000;16(11):1046-7.

77. Amiryousefi A, Hyvonen J, Poczai P. IRscope: an online program to visualize the junction sites of chloroplast genomes. Bioinformatics. 2018;34(17):3030-1

78. Bi G, Mao Y, Xing Q, Cao M. HomBlocks: A multiple-alignment construction pipeline for organelle phylogenomics based on locally collinear block searching. Genomics. 2018;110(1):18-22.

79. Castresana J. Selection of conserved blocks from multiple alignments for their use in phylogenetic analysis. Mol Biol Evol. 2000;17(4):540-52.

80 Guindon S, Dufayard JF, Lefort V, Anisimova M, Hordijk W, Gascuel O. New algorithms and methods to estimate maximum-likelihood phylogenies: assessing the performance of PhyML 3.0. Syst Biol. 2010;59(3):307-21.

81 Tamura K, Stecher G, Peterson D, Filipski A, Kumar S. MEGA6: molecular evolutionary genetics analysis version 6.0. Mol Biol Evol. 2013;30(12):2725-9.

\section{Publisher's Note}

Springer Nature remains neutral with regard to jurisdictional claims in published maps and institutional affiliations.

Ready to submit your research? Choose BMC and benefit from:

- fast, convenient online submission

- thorough peer review by experienced researchers in your field

- rapid publication on acceptance

- support for research data, including large and complex data types

- gold Open Access which fosters wider collaboration and increased citations

- maximum visibility for your research: over $100 \mathrm{M}$ website views per year

At BMC, research is always in progress.

Learn more biomedcentral.com/submissions 